\title{
MiR-200c promotes bladder cancer cell migration and invasion by directly targeting RECK
}

\author{
This article was published in the following Dove Press journal: \\ OncoTargets and Therapy \\ 16 August 2016 \\ Number of times this article has been viewed
}

\author{
Yidong Cheng* \\ Xiaolei Zhang* \\ Peng Li* \\ Chengdi Yang \\ Jinyuan Tang \\ Xiaheng Deng \\ Xiao Yang \\ Jun Tao \\ Qiang Lu \\ Pengchao Li
}

Department of Urology, The First Affiliated Hospital of Nanjing Medical University, Nanjing, People's Republic of China

*These authors contributed equally to this work
Correspondence: Qiang Lu; Pengchao Li Department of Urology, The First Affiliated Hospital of Nanjing Medical University, 300 Guangzhou Road, Nanjing 210029, Jiangsu Province, People's

Republic of China

Tel +8625 837l 0079

Email doctorlvqiang@sina.com; superkulian@aliyun.com
Background: Increasing evidence suggests that the dysregulation of certain microRNAs plays an important role in tumorigenesis and metastasis. MiR-200c exhibits a disordered expression in many tumors and presents dual roles in bladder cancer (BC). Therefore, the definite role of miR-200c in BC needs to be investigated further.

Materials and methods: Quantitative reverse transcription polymerase chain reaction was used to assess miR-200c expression. Cell invasion and migration were evaluated using wound healing and transwell assays. The luciferase reporter assay was used to identify the direct target of miR-200c. The expression of reversion-inducing cysteine-rich protein with kazal motifs $(R E C K)$ in $\mathrm{BC}$ tissues and adjacent nontumor tissues, as well as in $\mathrm{BC}$ cell lines, was detected through quantitative reverse transcription polymerase chain reaction, Western blot assay, and immunohistochemistry.

Results: The miR-200c expression was significantly upregulated in the BC tissues compared with the adjacent nontumor tissues. The downregulation of miR-200c significantly inhibited cell migration and invasion in the $\mathrm{BC}$ cell lines. The luciferase reporter assay showed that RECK was a direct target of miR-200c. The knockdown of RECK in the BC cell lines treated with anti-miR-200c elevated the previously attenuated cell migration and invasion.

Conclusion: Our findings indicated that miR-200c functions as oncogenes in $\mathrm{BC}$ and may provide a novel therapeutic strategy for the treatment of $\mathrm{BC}$.

Keywords: miR-200c, bladder cancer, migration, invasion, $R E C K$

\section{Introduction}

Bladder cancer $(\mathrm{BC})$ is the second most frequent malignancy of the urinary tract. $\mathrm{BC}$ can be classified as either a nonmuscle-invasive bladder cancer (NMIBC) or a muscle-invasive bladder cancer (MIBC). NMIBC (Ta, carcinoma in situ, and T1) that does not penetrate the basement membrane is rarely associated with patient death. ${ }^{1}$ However, MIBC ( $\geq \mathrm{T} 2$ ) is potentially life-threatening metastasis and has a different biology than NMIBC. Despite radical cystectomy and systemic therapy, including immunotherapy, chemotherapy, and radiotherapy, at least $50 \%$ of patients with MIBC die from metastases within 2 years of diagnosis., ${ }^{2,3}$ Therefore, exploring the potential mechanisms of $\mathrm{BC}$ migration and invasion is urgently needed.

MicroRNAs (miRNAs) are a class of small noncoding ( $\sim 18-22$ nucleotides) RNAs that negatively regulate the expression of target genes by binding to the 3 '-untranslated region ( $3^{\prime}$-UTR $) .{ }^{4}$ Growing evidence reveals that the disordered expression of miRNA is related to human cancer initiation and progression, including that of miR-21 in prostate cancer, miR-145 in lung cancer, and miR-140 in breast cancer. ${ }^{5-7}$ In the recent years, the aberrant regulation of miRNAs in $\mathrm{BC}$ has been suggested to contribute to bladder tumorigenesis. ${ }^{8}$ Therefore, the identification of miRNAs in BC is critical. 
MiR-200c belongs to the miRNA-200 family and is located on chromosome $12 \mathrm{p} 13$. The majority of research has shown that miR-200c is deregulated and acts as a tumor suppressor in several tumors. However, the role of miR-200c in BC remains inconsistent. Therefore, we present this study to understand the specific function of miR-200c in BC.

In this study, we investigated the miR-200c expression in BC tissues. We studied the effects of miR-200c on cell migration and invasion in $\mathrm{BC}$ cell lines. Moreover, reversion-inducing cysteine-rich protein with kazal motifs $(R E C K)$, a tumor suppressor gene, was identified as a target of miR-200c. Our results suggested that miR-200c could promote cell migration and invasion by targeting $R E C K$ in $\mathrm{BC}$ cells.

\section{Materials and methods}

\section{Clinical specimens and cell lines}

A total of 22 paired $\mathrm{BC}$ tissues and adjacent nontumor tissues were obtained from patients undergoing radical cystectomy at the First Affiliated Hospital of Nanjing Medical University. A total of 14 paired tissue samples were immediately frozen in liquid nitrogen, and eight paired tissue samples were fixed in formalin. All patients provided their signed informed consent, and this study was approved by the Research Ethics Committee of the First Affiliated Hospital of Nanjing Medical University. The tumor stage was determined according to the 2004 World Health Organization classification.

The human BC cell lines T24 and EJ were obtained from the Type Culture Collection of the Chinese Academy of Sciences (Shanghai, People's Republic of China) and were cultured in Roswell Park Memorial Institute 1640 medium (Thermo Fisher Scientific, Waltham, MA, USA) with 10\% fetal bovine serum (Thermo Fisher Scientific), $100 \mathrm{IU} / \mathrm{mL}$ penicillin, and $100 \mu \mathrm{g} / \mathrm{mL}$ streptomycin. 293T cell was cultured in Dulbecco's Modified Eagle's Medium with 10\% fetal bovine serum. All cells were maintained in a humidified incubator at $37^{\circ} \mathrm{C}$ with $5 \% \mathrm{CO}_{2}$.

\section{RNA isolation and quantitative reverse transcription polymerase chain reaction}

Total RNA was extracted from tissue samples and cell lines using miRNeasy Mini Kit (Qiagen NV, Venlo, the Netherlands). RNA was used to synthesize complementary DNA using Mir-X miRNA First-Strand Synthesis Kit (Clontech Laboratories, Inc., USA) for miRNA quantity and PrimeScript $^{\mathrm{TM}}$ RT Master Mix (Takara Bio Inc., Japan) for messenger RNA (mRNA) quantity. The levels of miRNA and mRNA were determined through quantitative reverse transcription polymerase chain reaction (qRT-PCR) using SYBR Green Master Mix (Takara Bio Inc., Japan). The primers for Takara Bio Inc., Japan were forward 5'-CCT CAG TGA GCA CAG TTC AGA-3' and reverse 5'-GCA GCA CAC ACA CTG CTG TA-3' and for $\beta$-actin were forward 5'-AGC GAG CAT CCC CCA AAG TT-3' and reverse 5'-GGG CAC GAA GGC TCA TCA TT-3' (Sangon Biotech, Shanghai, People's Republic of China). U6 and $\beta$-actin were used as internal controls for miRNA and mRNA quantification. All the reactions were performed in triplicate.

\section{Oligonucleotides transfection}

MiR-200c mimics, anti-miR-200c, small interfering RNA of RECK ( $s i R E C K$ ), and their corresponding negative controls (miR-NC, anti-miR-NC, and siNC) were purchased from GenePharma (Shanghai, People's Republic of China). T24 and EJ cells were transfected with oligonucleotides using Lipofectamine 2000 reagent (Thermo Fisher Scientific, Waltham, MA, USA) according to the manufacturer's instructions.

\section{Wound healing assay}

When the cells reached $95 \%$ confluence in six-well plates after transfection, a $200 \mu \mathrm{L}$ pipette tip was used to draw a wound with the same width. After washing and removing cell debris, the cells were cultured using a serum-free medium under standard conditions. The wound was observed under a microscope (Olympus Corporation, Tokyo, Japan) at 40× magnification at two preselected time points ( 0 and 24 hours). The widths of the wounds were counted.

\section{Migration and invasion assays}

Cell migration and invasion were determined using a transwell chamber ( $8 \mu \mathrm{m}$ pore size) with and without $\mathrm{BD}$ Matrigel (BD Biosciences, San Jose, CA, USA). The upper side of the membrane was coated with Matrigel for the invasion assay. After 24 hours of transfection, a total of $2 \times 10^{4}$ cells in $200 \mu \mathrm{L}$ serum-free medium were plated to the upper chambers and $600 \mu \mathrm{L}$ medium containing $10 \%$ serum was used as a chemoattractant in the lower chambers. After 48 hours, the cells on the upper side of the membrane were removed using cotton swabs, and the invaded cells on the lower side of the membrane were fixed, stained, and counted using an inverted microscope at $100 \times$ magnification.

\section{Western blot analysis}

The cells were lysed after 48 hours of transfection. Their protein was extracted using radioimmunoprecipitation assay buffer and was quantified using a bicinchonininc acid 
(BCA) Protein Assay Kit (Beyotime, Shanghai, People's Republic of China). Equal amounts of proteins were separated through $10 \%$ sodium dodecyl sulfate polyacrylamide gel electrophoresis and were transferred to polyvinylidene difluoride membranes. The membranes were blocked with 5\% nonfat milk and were incubated with primary antibodies against RECK (1:1,000; Cell Signaling Technology, Inc., USA), matrix metalloproteinase-2 (MMP-2; 1:1,000; Cell Signaling Technology), and glyceraldehyde 3-phosphate dehydrogenase (Cell Signaling Technology) overnight at $4^{\circ} \mathrm{C}$. After washing with Tris-buffered saline containing Tween 20, the membranes were incubated with secondary antirabbit or antimouse antibodies for 1 hour at room temperature and were subsequently detected using a chemiluminescence system (Bio-Rad Laboratories Inc., Hercules, CA).

\section{Bioinformatic and luciferase reporter assay}

Mirorna.org, miRBase, and TargetScan were used to predict the potential targets of miR-200c. 293T cells were cotransfected with miR-200c mimics or miR-mimic controls and with wild-type or mutant-type plasmid containing pEZX/RECK-1-3'-UTR or pEZX/RECK-1-3'-UTR, respectively, together with firefly and Renilla luciferase (GeneCopoeia, Rockville, MD) using Lipofectamine 2000. The luciferase activity was determined by using the Luc-Pair miR Luciferase Assay (GeneCopoeia) after 48 hours of cotransfection according to the manufacturer's instructions.

\section{Immunohistochemical staining}

Formalin-fixed tissue samples were embedded in paraffin and cut into $4 \mu \mathrm{m}$ sections. Immunohistochemistry staining was performed as previously described..$^{9}$ The RECK expression was defined as low and high based on the RECK staining intensity of the cells.

\section{Statistical analyses}

The results were expressed as mean \pm standard deviation and were analyzed using SPSS 16.0 (SPSS Inc., Chicago, IL). All statistical analyses were performed using the SPSS 16.0 software. Data were analyzed using a two-tailed Student's $t$-test or one-way analysis of variance. $P<0.05$ was considered statistically significant.

\section{Results \\ MiR-200c is upregulated in BC clinical specimens}

To explore the role of miR-200c in BC, we first compared the miR-200c expression in the BC clinical specimens through qRT-PCR. The result showed that the miR-200c expression significantly increased in $\mathrm{BC}$ tissues compared with their paired adjacent nontumor tissues (Figure 1A). To further explore the miR-200c expression in BC tissues, we divided the 14 patients into two groups according to postoperative pathology: $<\mathrm{T} 2(\mathrm{n}=7)$ and $\geq \mathrm{T} 2$ ( $\mathrm{n}=7$ ). Interestingly, the miR-200c expression in MIBC was significantly higher than that in NMIBC (Figure 1B).

To understand the role of $\mathrm{miR}-200 \mathrm{c}$ in cell biological functions better, both invasive BC cell lines (T24 and EJ) were transfected with anti-miR-200c and anti-miR-NC. The ectopic expression of miR-200c was confirmed through qRT-PCR (Figure 1C).

\section{MiR-200c promotes BC cell migration and invasion}

To further confirm the function of miR-200c in BC cell lines, we transfected BC cell lines EJ and T24 with anti-miR-200c and anti-miR-NC. The wound healing assay demonstrated that the knockdown of miR-200c in BC cells significantly
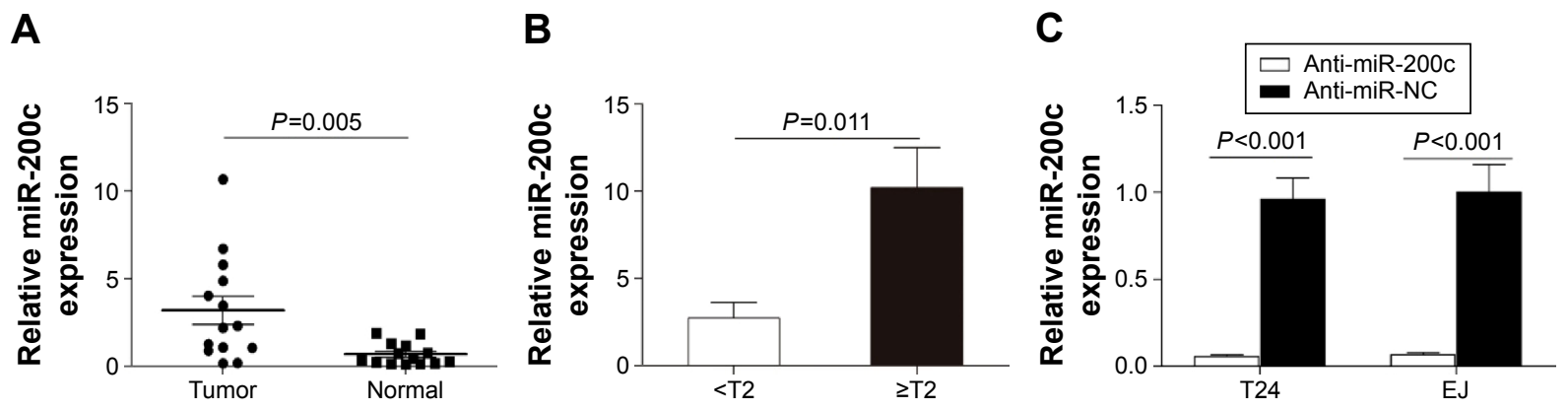

Figure I Expression of miR-200c in tissues and BC cell lines.

Notes: (A) The relative expression of miR-200c in 14 paired BC tissues was significantly higher than that in the normal tissues. (B) The relative expression of miR-200c in MIBC ( $\geq T 2$ ) was much higher than that in NMIBC ( $<$ T2). (C) Downregulation of the miR-200c expression in BC T24 and EJ cell lines after transfection with anti-miR-200c compared with the corresponding controls.

Abbreviations: BC, bladder cancer; MIBC, muscle-invasive bladder cancer; NMIBC, nonmuscle-invasive bladder cancer; NC, negative control. 


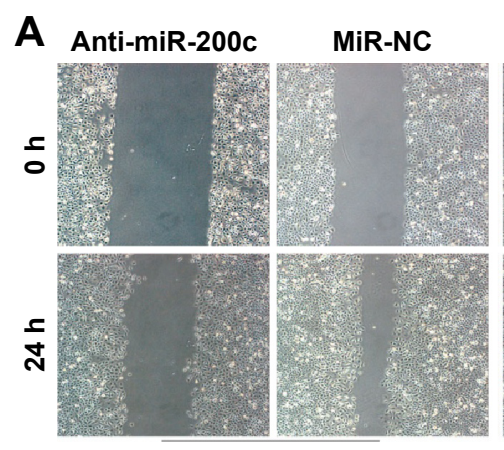

T24

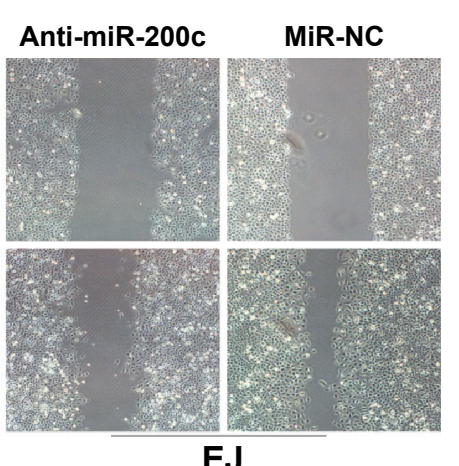

EJ

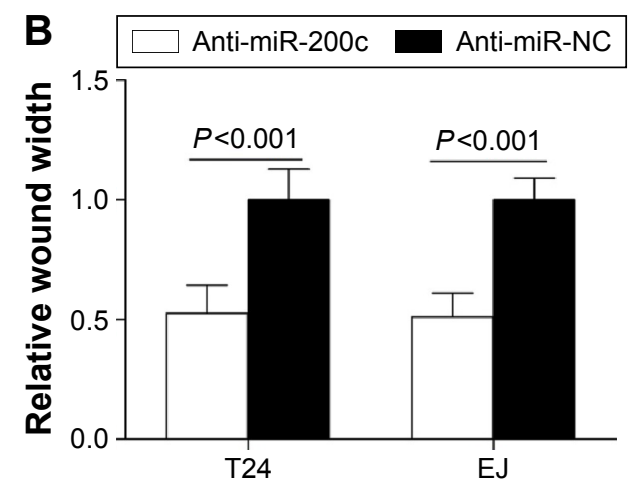

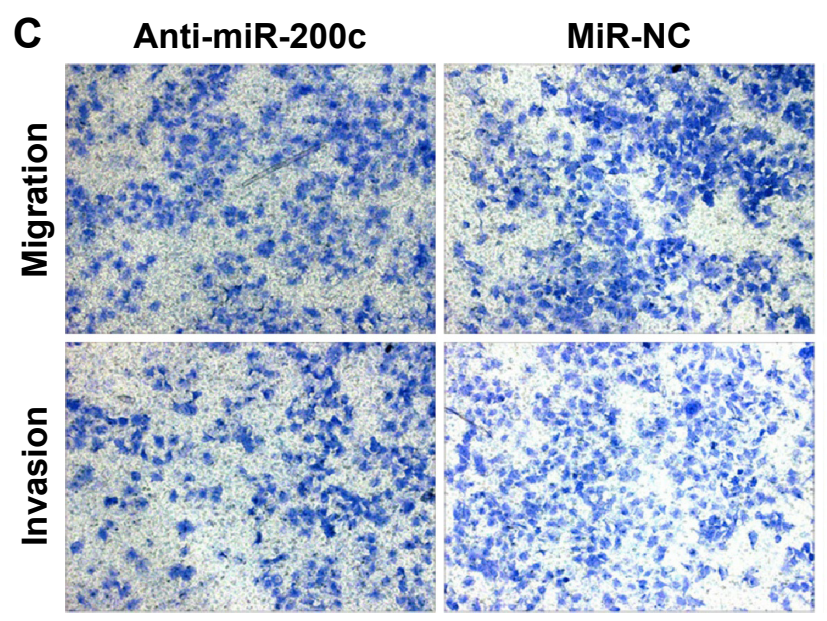

T24

D



Anti-miR-200c

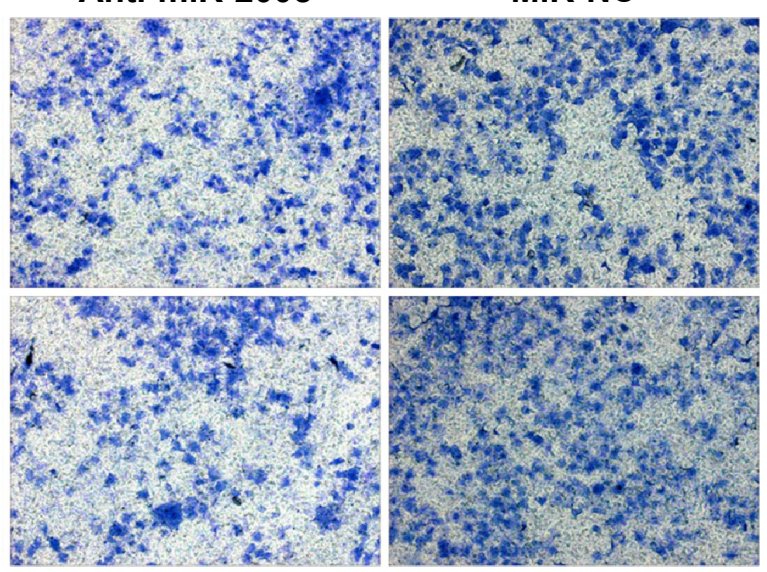

EJ

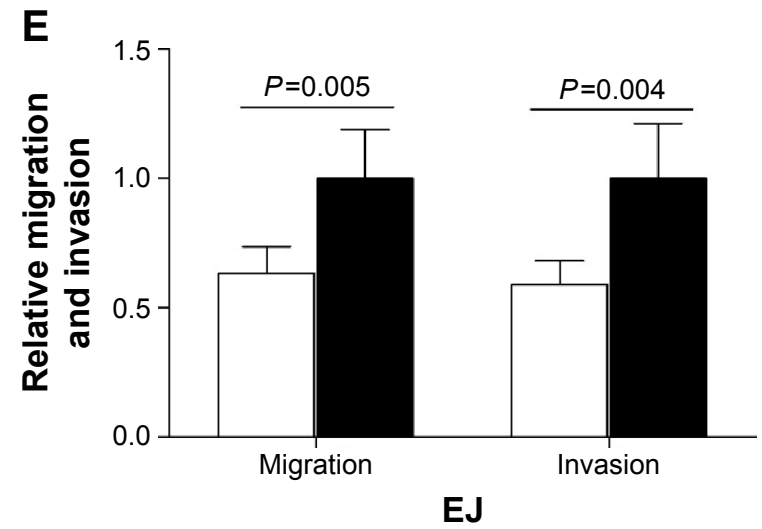

Anti-miR-200c

Anti-miR-NC

Figure 2 MiR-200c promoted the migration and invasion of $B C$ cell lines.

Notes: (A) Representative images of the wound healing assay. (B) Anti-miR-200c significantly inhibited the motility of BC T24 and EJ cells compared with the control. (C) Representative images of the transwell assay. (D and E) Anti-miR-200c significantly inhibited the cell migration and invasion of BC T24 and EJ cells compared with the control.

Abbreviations: BC, bladder cancer; NC, negative control; h, hours.

decreased the mobility of BC cells (Figure $2 \mathrm{~A}$ and B). Similarly, the migration and invasion assays showed that antimiR-200c significantly inhibited the migratory and invasive abilities of BC cells but not those of the control (Figure 2C-E). These results suggested that miR-200c significantly contributed to the migration and invasion of $\mathrm{BC}$ cells.

\section{MiR-200c directly regulates RECK}

We found that $R E C K$ could be a potential target of miR-200c, and this finding was confirmed in our study through bioinformatics analysis (Figure 3A). Based on the publicly available algorithms, the $3^{\prime}$-UTR of the RECK gene contains two complementary sites for miR-200c. We then performed 


\section{A}

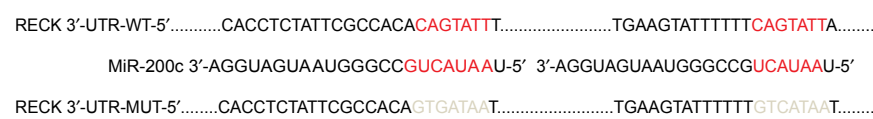

B

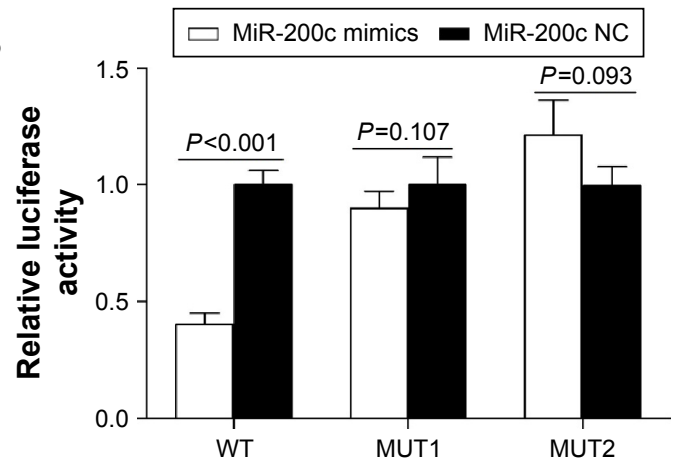

C

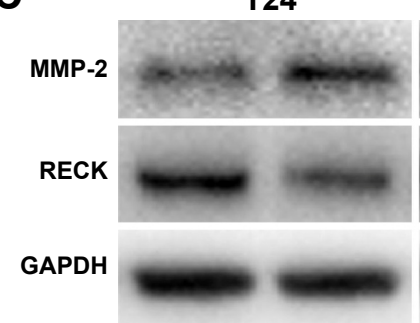

Anti-miR-200c Anti-miR-NC Anti-miR-200c Anti-miR-NC

D



Figure 3 RECK is a direct target of miR-200c in BC cell lines.

Notes: (A) A schematic of the putative miR-200c binding sites and the corresponding mutants on RECK $3^{\prime}$-UTR. (B) Dual luciferase reporter assays demonstrated that miR200c overexpression could reduce the fluorescence intensity in 293T cells cotransfected with miR-200c mimics or control and the wild-type RECK $3^{\prime}$-UTR vector (WT), whereas miR-200c did not exert an effect on the mutant-type RECK 3'-UTR vector (Mut). (C) Representative Western blot images of RECK and MMP-2 proteins. GAPDH was used as the internal control. (D) Downregulation of miR-200c by anti-miR-200c increased the RECK protein level and subsequently reduced MMP-2 expression.

Abbreviations: RECK, reversion-inducing cysteine-rich protein with kazal motifs; BC, bladder cancer; UTR, untranslated region; MMP-2, matrix metalloproteinase-2; GAPDH, glyceraldehyde 3-phosphate dehydrogenase; NC, negative control.

a dual luciferase reporter assay to test the interaction between miR-200c and the 3'-UTR of RECK. 293T cells were cotransfected with either miR-200c or miR-NC and a luciferase reporter comprising the wild-type RECK 3 '-UTR or the mutant RECK 3'-UTR. The luciferase reporter assay showed that miR-200c significantly suppressed the luciferase activity of the wild-type $R E C K 3^{\prime}$-UTR without affecting the mutant RECK 3'-UTR (Figure 3B).

Western blot assay also confirmed that the RECK protein levels significantly increased in anti-miR-200c-transfected $\mathrm{BC}$ cells but not in those transfected with anti-miR-NC (Figure 3C and D). These findings indicated that miR-200c directly targets $R E C K 3^{\prime}$-UTR and negatively regulates its expression.

\section{Silencing of RECK promotes migration and invasion of $B C$ cells}

To further determine miR-200c-regulated cell migration and invasion in BC cells by modulating RECK, we cotransfected EJ and T24 cell lines with siRECK and anti-miR-200c. The RECK protein levels were determined through Western blot assay (Figure 4A and B). The downregulation of RECK treated with siRECK was evidently counteracted by anti-miR200c. Moreover, cell migration and invasion were confirmed by the wound healing and transwell assays. As expected, a reduction in RECK protein levels increased the migration and invasion of EJ and T24 cell lines (Figure 4C-G). Conversely, the increased $R E C K$ expression suppressed the migratory and invasive ability of EJ and T24 cell lines (Figure 4C-G). These results showed that the migratory and invasive abilities of $\mathrm{BC}$ cells decreased as $R E C K$ expression increased and suggested that miR-200c promoted the migration and invasion of $B C$ cells through the miR-200c/RECK signaling axis.

\section{RECK is downregulated in $B C$ clinical specimens}

The qRT-PCR analysis demonstrated that the RECK mRNA level was markedly reduced in the BC tissues but not in the normal tissues (Figure 5A). Immunohistochemistry assays on bladder tissues showed low RECK protein expression through the weak staining in the tumor tissues compared with the normal tissues (Figure 5B). In addition, RECK was detected in the cytoplasm and plasma membrane of normal $\mathrm{BC}$ cells, consistent with the results of other studies. ${ }^{10,11}$ 
A

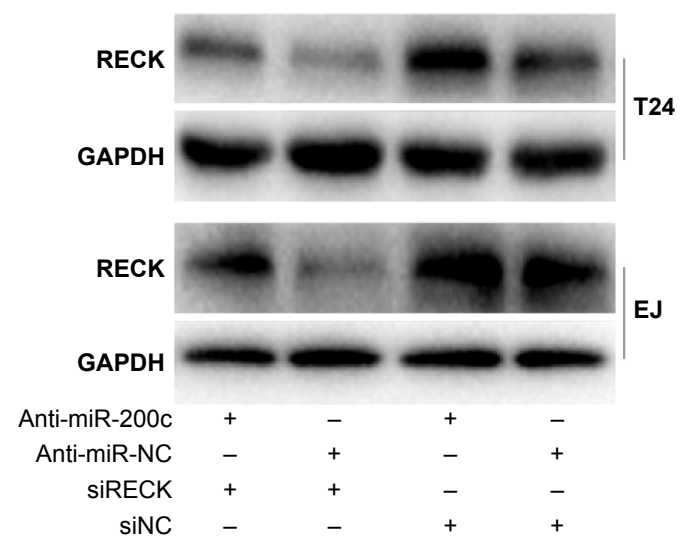

C

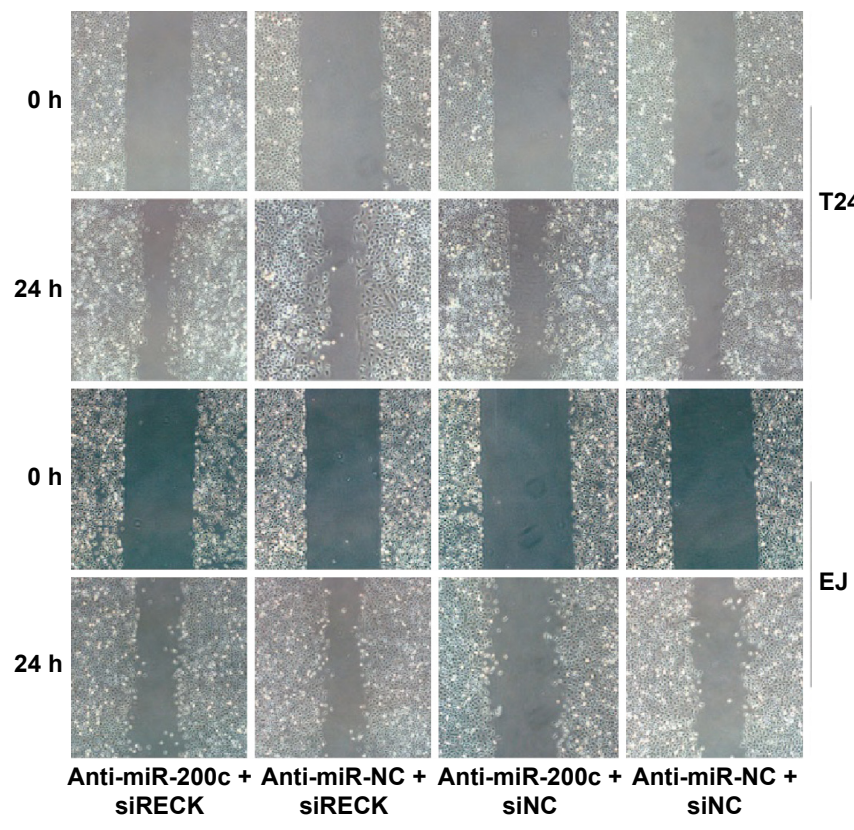

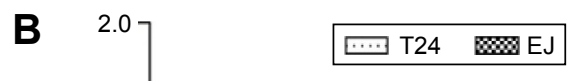
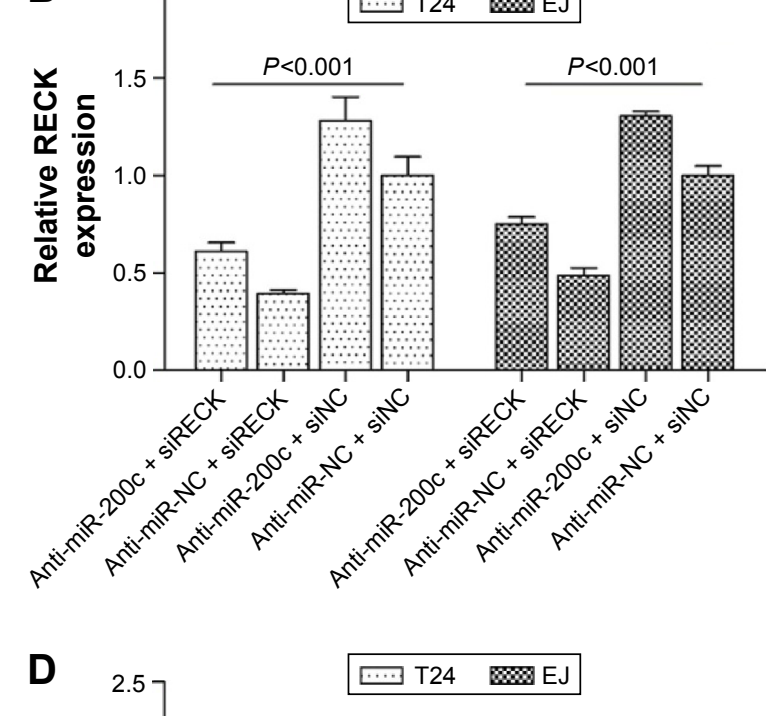

T24

EJ
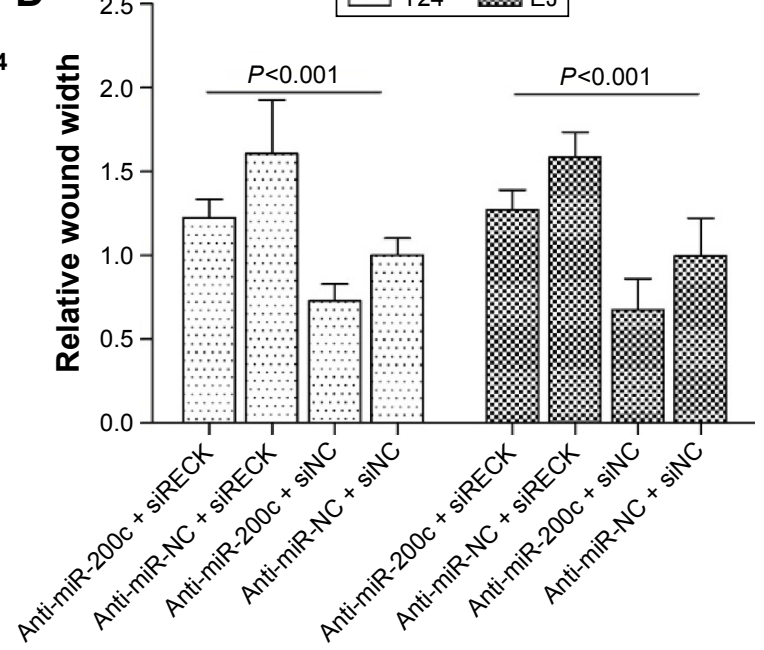



Figure 4 (Continued) 




Figure 4 Repression of RECK rescues the anti-miR-200c-associated increase in cell migration and invasion of BC cell lines.

Notes: (A and B) Western blot detection of the RECK protein expression level in T24 cells or EJ cells cotransfected with anti-miR-200c or anti-miR-NC and siRECK or siNC. (C and D) Wound healing assay analysis of the migration and invasion in T24 cells or EJ cells cotransfected with anti-miR-200c or anti-miR-NC and siRECK or siNC. (E-G) Transwell assay analysis of the migration and invasion in T24 cells or EJ cells cotransfected with anti-miR-200c or anti-miR-NC and siRECK or siNC.

Abbreviations: RECK, reversion-inducing cysteine-rich protein with kazal motifs; BC, bladder cancer; NC, negative control; siRNA, small interfering RNA; siRECK, siRNA of RECK; GAPDH, glyceraldehyde 3-phosphate dehydrogenase; h, hours.

\section{Discussion}

Metastasis is responsible for as much as $90 \%$ of cancerrelated mortality, but it remains the most poorly understood component of cancer pathogenesis. ${ }^{12}$ The migration and invasion of cancer cells may be the key steps in this complex multistep process through which cancer cells disseminate and spread from a primary tumor to distant sites, forming secondary tumors in other organs and tissues. ${ }^{13,14}$ Increasing current evidence suggests that aberrant miRNA expression contributes to invasion and metastasis. ${ }^{15,16}$ miRNAs have already been known to be involved in the initiation, development, and metastasis of BC, and several of them can function as oncogenes or as tumor suppressors by regulating the expression of their target genes. ${ }^{17}$ The miR-200 family is involved in the epithelial-to-mesenchymal transition, tumor cell invasion and metastasis, and self-renewal of cancer stem cells. ${ }^{18}$ miR-200c, as the prevailing member of the miRNA-200 family, has been demonstrated to be important in modulating tumor biology. Currently, miR-200c has been reported to be upregulated in melanoma and ovarian cancer ${ }^{19,20}$ and downregulated in renal cell carcinoma and gastric cancer, ${ }^{21,22}$ functioning as an oncogene and a tumor suppressor, respectively. Consequently, the dual roles of miR-200c may occur in different types of cancer in a tissuespecific manner. Nevertheless, miR-200c has been reported to be upregulated in $\mathrm{BC},{ }^{23,24}$ whereas others have reported it to be downregulated. ${ }^{25}$ These contradictory findings may be in part due to the different models applied in these studies.
A

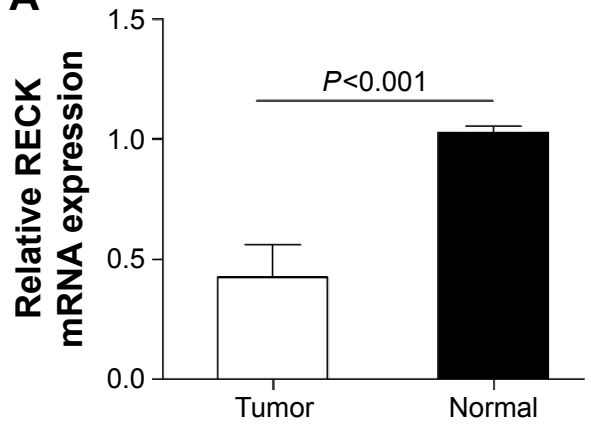

B



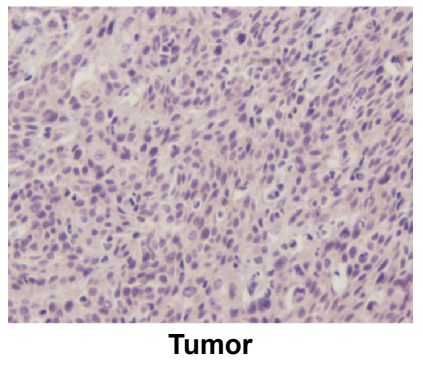

Figure 5 Expression of RECK in tissues.

Notes: (A) The relative expression of RECK mRNA in BC tissues was significantly lower than that in the normal tissues. (B) The expression of RECK protein was analyzed using immunohistochemistry.

Abbreviations: RECK, reversion-inducing cysteine-rich protein with kazal motifs; mRNA, messenger RNA; BC, bladder cancer. 
Therefore, the roles of miR-200c in BC are also ambiguous, and its definite function needs to be further investigated.

To affirm the role of miR-200c in BC in more detail, we first ascertained the expression of miR-200c in BC specimens. We found that miR-200c expression was upregulated in $\mathrm{BC}$ but not in adjacent nontumor tissues. Moreover, increased miR-200c expression was observed in MIBC but not in NMIBC. Subsequently, we selected the two invasive BC cell lines T24 and EJ for the miR-200c loss-of-function assays. We found that the knockdown of miR-200c in T24 and EJ cell lines resulted in decreased migratory and invasive abilities, thus indicating the migratory and invasive promoter role of miR-200c in $\mathrm{BC}$.

Identifying the downstream target genes of miR-200c can contribute to the understanding of its potential molecular mechanisms. We used bioinformatics analysis to search for potential targets of miR-200c. $R E C K$, a gene previously implicated in metastasis and invasiveness, ${ }^{10,26}$ was identified to be the highest possible target of miR-200c. As a membrane-anchored glycoprotein, the RECK protein mainly functions in tissue remodeling and inhibits the activity of MMPs. ${ }^{27,28}$ MMPs proteolytically degrade extracellular matrix proteins, which are critical for tumor metastasis and invasion. Moreover, Wittschieber et $\mathrm{al}^{11}$ reported that decreased RECK expression is associated with increasing stage and grade of $\mathrm{BC}$ and could serve as a molecular marker for the distinction between NMIBC and MIBC. As a member of the MMPs, MMP-2 evidently decreased and RECK significantly increased in the anti-miR-200c group in this study (Figure 3C). These results were consistent with those of other research. ${ }^{27,29}$ Therefore, our hypothesis is that miR-200c promotes BC cell migration and invasion by targeting RECK through the inhibition of MMP-2 expression. We demonstrated that miR-200c directly bound the RECK $3^{\prime}$-UTR and that the knockdown of miR-200c increased the RECK protein expression. However, the unchanged RECK mRNA level could have been due to the posttranscriptional regulation (data not shown). Subsequently, the knockdown of RECK with siRNA induced a significant increase in the migration and invasion abilities of BC cells. Thus, we suggested that miR-200c negatively regulated the expression of $R E C K$ and affected the biological processes of $\mathrm{BC}$.

\section{Conclusion}

In summary, our study revealed that miR-200c is overexpressed and acts as an oncogene in BC. To the best of our knowledge, this is the first study to demonstrate that miR-200c promotes the migration and invasion of BC cells through the downregulation of MMPs by targeting RECK. Consequently, the identification of miR-200c may help us understand the potential molecular mechanisms of BC tumorigenesis and may provide a novel therapeutic strategy for the treatment of $\mathrm{BC}$.

\section{Acknowledgments}

This work was supported by the National Natural Science Foundation of China (grant nos 81201997 and 81272832) and the Priority Academic Program Development of Jiangsu Higher Education Institutions and funded by the Jiangsu Provincial Special Program of Medical Science (BL2012027). The funders had no role in the study design, data collection and analysis, decision to publish, or preparation of the manuscript.

\section{Disclosure}

The authors report no conflicts of interest in this work.

\section{References}

1. Gallagher DJ, Milowsky MI. Bladder cancer. Curr Treat Options Oncol. 2009;10(3-4):205-215.

2. Liebert M, Seigne J. Characteristics of invasive bladder cancers: histological and molecular markers. Semin Urol Oncol. 1996;14(2): $62-72$.

3. Steinberg GD, Trump DL, Cummings KB. Metastatic bladder cancer. Natural history, clinical course, and consideration for treatment. Urol Clin North Am. 1992;19(4):735-746.

4. Bartel DP. MicroRNAs: target recognition and regulatory functions. Cell. 2009;136(2):215-233.

5. Ribas J, Ni X, Haffner M, et al. miR-21: an androgen receptor-regulated microRNA that promotes hormone-dependent and hormone-independent prostate cancer growth. Cancer Res. 2009;69(18):7165-7169.

6. Cho WC, Chow AS, Au JS. Restoration of tumour suppressor hsa-miR145 inhibits cancer cell growth in lung adenocarcinoma patients with epidermal growth factor receptor mutation. Eur J Cancer. 2009;45(12): 2197-2206.

7. Li Q, Yao Y, Eades G, Liu Z, Zhang Y, Zhou Q. Downregulation of miR-140 promotes cancer stem cell formation in basal-like early stage breast cancer. Oncogene. 2014;33(20):2589-2600.

8. Catto JW, Alcaraz A, Bjartell AS, et al. MicroRNA in prostate, bladder, and kidney cancer: a systematic review. Eur Urol. 2011;59(5): 671-681.

9. Cheng Y, Yang X, Deng X, et al. MicroRNA-218 inhibits bladder cancer cell proliferation, migration, and invasion by targeting BMI-1. Tumour Biol. 2015;36(10):8015-8023.

10. Takeuchi T, Hisanaga M, Nagao M, et al. The membrane-anchored matrix metalloproteinase (MMP) regulator RECK in combination with MMP-9 serves as an informative prognostic indicator for colorectal cancer. Clin Cancer Res. 2004;10(16):5572-5579.

11. Wittschieber D, Stenzinger A, Klauschen F, et al. Decreased RECK and Increased EMMPRIN expression in urothelial carcinoma of the bladder are associated with tumor aggressiveness. Pathobiology. 2011;78(3): 123-131.

12. Chaffer CL, Weinberg RA. A perspective on cancer cell metastasis. Science. 2011;331(6024):1559-1564.

13. Zhou L, Liu F, Wang X, Ouyang G. The roles of microRNAs in the regulation of tumor metastasis. Cell Biosci. 2015;5:32. 
14. Wan L, Pantel K, Kang Y. Tumor metastasis: moving new biological insights into the clinic. Nat Med. 2013;19(11):1450-1464.

15. White NM, Fatoohi E, Metias M, Jung K, Stephan C, Yousef GM. Metastamirs: a stepping stone towards improved cancer management. Nat Rev Clin Oncol. 2011;8(2):75-84.

16. Aigner A. MicroRNAs (miRNAs) in cancer invasion and metastasis: therapeutic approaches based on metastasis-related miRNAs. $J$ Mol Med. 2011;89(5):445-457.

17. Yoshino H, Seki N, Itesako T, Chiyomaru T, Nakagawa M, Enokida H. Aberrant expression of microRNAs in bladder cancer. Nat Rev Urol. 2013;10(7):396-404.

18. Feng X, Wang Z, Fillmore R, Xi Y. MiR-200, a new star miRNA in human cancer. Cancer Lett. 2014;344(2):166-173.

19. Elson-Schwab I, Lorentzen A, Marshall CJ. MicroRNA-200 family members differentially regulate morphological plasticity and mode of melanoma cell invasion. PLoS One. 2010;5(10):e13176.

20. Iorio MV, Visone R, Di Leva G, et al. MicroRNA signatures in human ovarian cancer. Cancer Res. 2007;67(18):8699-8707.

21. Chang I, Mitsui Y, Fukuhara S, et al. Loss of miR-200c up-regulates CYP1B1 and confers docetaxel resistance in renal cell carcinoma. Oncotarget. 2015;6(10):7774-7787.

22. Zhou X, Wang Y, Shan B, et al. The downregulation of miR-200c/141 promotes ZEB1/2 expression and gastric cancer progression. Med Oncol. 2015;32(1):428.
23. Xie $\mathrm{P}, \mathrm{Xu} \mathrm{F}$, Cheng $\mathrm{W}$, et al. Infiltration related miRNAs in bladder urothelial carcinoma. J Huazhong Univ Sci Technolog Med Sci. 2012; 32(4):576-580.

24. Han Y, Chen J, Zhao X, et al. MicroRNA expression signatures of bladder cancer revealed by deep sequencing. PLoS One. 2011;6(3):e18286.

25. Liu L, Qiu M, Tan G, et al. miR-200c Inhibits invasion, migration and proliferation of bladder cancer cells through down-regulation of BMI-1 and E2F3. $J$ Transl Med. 2014;12(1):305.

26. Masui T, Doi R, Koshiba T, et al. RECK expression in pancreatic cancer: its correlation with lower invasiveness and better prognosis. Clin Cancer Res. 2003;9(5):1779-1784.

27. Alexius-Lindgren M, Andersson E, Lindstedt I, Engstrom W. The RECK gene and biological malignancy - its significance in angiogenesis and inhibition of matrix metalloproteinases. Anticancer Res. 2014;34(8): 3867-3873.

28. Takahashi C, Sheng Z, Horan TP, et al. Regulation of matrix metalloproteinase- 9 and inhibition of tumor invasion by the membraneanchored glycoprotein RECK. Proc Natl Acad Sci U S A. 1998;95(22): 13221-13226.

29. Bai T, Dong DS, Pei L. Synergistic antitumor activity of resveratrol and miR-200c in human lung cancer. Oncol Rep. 2014;31(5):2293-2297.
OncoTargets and Therapy

\section{Publish your work in this journal}

OncoTargets and Therapy is an international, peer-reviewed, open access journal focusing on the pathological basis of all cancers, potential targets for therapy and treatment protocols employed to improve the management of cancer patients. The journal also focuses on the impact of management programs and new therapeutic agents and protocols on

\section{Dovepress}

patient perspectives such as quality of life, adherence and satisfaction. The manuscript management system is completely online and includes a very quick and fair peer-review system, which is all easy to use. Visit http://www.dovepress.com/testimonials.php to read real quotes from published authors. 